\section{PATHOLOGIC COMPARISON OF VIDEO-ASSISTED THORACIC SURGICAL LUNG BIOPSY WITH TRADITIONAL OPEN LUNG BIOPSY}

From the Department of Laboratory Medicine and Pathology and Division of Thoracic Surgery,' Mayo Clinic and Foundation, Rochester, Minn.

Received for publication March 24, 1994.

Accepted for publication August 29, 1994.

Address for reprints: Thomas V. Colby, MD, Department of Laboratory Medicine and Pathology, Mayo Clinic Scottsdale, 13400 East Shea Blvd., Scottsdale, AZ 85259.

Copyright (C) 1995 by Mosby-Year Book, Inc. $0022-5223 / 95 \$ 3.00+0 \quad \mathbf{1 2 / 1 / 6 0 3 3 5}$
Video-assisted thoracic surgical lung biopsy is an alternative to traditional open lung biopsy for diagnosis in patients with pleuropulmonary diseases. Between January 7, 1991, and August 3, 1993, 71 consecutive patients had video-assisted thoracic surgical lung biopsy and 42 patients had traditional open lung biopsy. A specific histologic diagnosis that correlated with the clinical findings was sought in each case and the yield was compared between the two groups. Procedure-related artifactual changes were also evaluated; the extent of traumatic hemorrhage and neutrophil margination as a result of tissue manipulation was significantly greater for patients in the video-assisted thoracic surgical lung biopsy group than for those in the open lung biopsy group, but the changes were generally minor and did not affect diagnostic yield. Complications developed in $11(15 \%)$ of 71 patients in the video-assisted thoracic surgical lung biopsy group including 5 patients with prolonged air leakage (more than 10 days); 2 with pneumonia; and 1 each with bleeding, late pneumothorax necessitating readmission, mucus plug necessitating bronchoscopy, and a hypoxic episode necessitating mechanical ventilation. On the other hand, 7 (17\%) of 42 patients in the open lung biopsy group had complications including 4 patients with prolonged air leakage (more than 10 days) and 3 with pneumonia. There were $6(8 \%)$ operative deaths in patients who had videoassisted thoracic surgical lung biopsy and $7(17 \%)$ in the open lung biopsy group; all had preoperative respiratory failure. We conclude that video-assisted thoracic surgical lung biopsy is an acceptable alternative to open lung biopsy for diagnosis of pulmonary infiltrates or indeterminate nodules. (J THORAC CARDIOvasC SURG 1995;109:494-8)

Mitsutaka Kadokura, MD, ${ }^{\mathrm{a}}$ Thomas V. Colby, MD, ${ }^{\mathrm{a}}$ Jeffrey L. Myers, MD, ${ }^{\mathrm{a}}$ Mark S. Allen, MD, ${ }^{\mathrm{b}}$ Claude Deschamps, MD, ${ }^{\mathrm{b}}$ Victor $\mathrm{F}$. Trastek, MD, ${ }^{\mathrm{b}}$ and Peter C. Pairolero, MD, ${ }^{\mathrm{b}}$ Rochester, Minn.
V ideo-assisted thoracic surgery is rapidly replacing standard thoracotomy in the evaluation and management of many pleuropulmonary diseases. Video-assisted thoracic surgical lung biopsy (VATSLB) is becoming the procedure of choice for the diagnosis of many localized and diffuse lung diseases and an adjunct to conventional bronchoscopic evaluation.

Anecdotally, we have noted a greater frequency of artifactual changes in the lung tissue obtained by VATS-LB in comparison with that obtained by open lung biopsy (OLB). In addition, we postulated that VATS-LB might offer less exposure and opportunity for the surgeon to determine the most appropriate site for biopsy resulting in a greater likelihood of sampling error. For these reasons, we reviewed a consecutive series of VATS-LB procedures and compared their diagnostic yield with a series of OLB procedures done during the same period.

\section{Patients and methods}

One hundred sixteen consecutive patients were referred to the Section of General Thoracic Surgery, Mayo Clinic, for lung biopsy to evaluate pulmonary abnormalities (primarily diffuse disease) between January 7, 1991, and August 3,1993. These patients form the basis of this study. The surgical approaches for VATS-LB and OLB have been previously described. ${ }^{1,2}$ There were 74 patients in the VATS-LB group; three were excluded because the pleural space was sufficiently obliterated by adhesions that the VATS-LB approach was abandoned and thoracotomy was necessary. The 71 patients who underwent VATS-LB because of parenchymal lung disease included 37 men and 34 women with a mean age of 56.9 years (range 16 to 86 ). Forty-two patients had traditional OLB: 27 men and 15 women with a mean age of 56.4 years (range 20 to 81 ).

In general, OLB was used instead of VATS-LB if patients were already intubated, had severe pulmonary dysfunction precluding one-lung ventilation, or had pleural adhesions that would prevent insertion of the thoracoscope. Double-lumen endotracheal anesthesia is necessary for VATS-LB because collapse of the ipsilateral lung is necessary for visualization of the pleural cavity. Conversely, complete collapse is not needed in OLB, and single-lumen endotracheal anesthesia is used.

Biopsy sites were selected on the basis of preoperative chest roentgenogram and computed tomography. One to three stapled wedge excisions of lung parenchyma were taken in each case. Specimens were serially sectioned and examined grossly in the fresh state before fixation in 


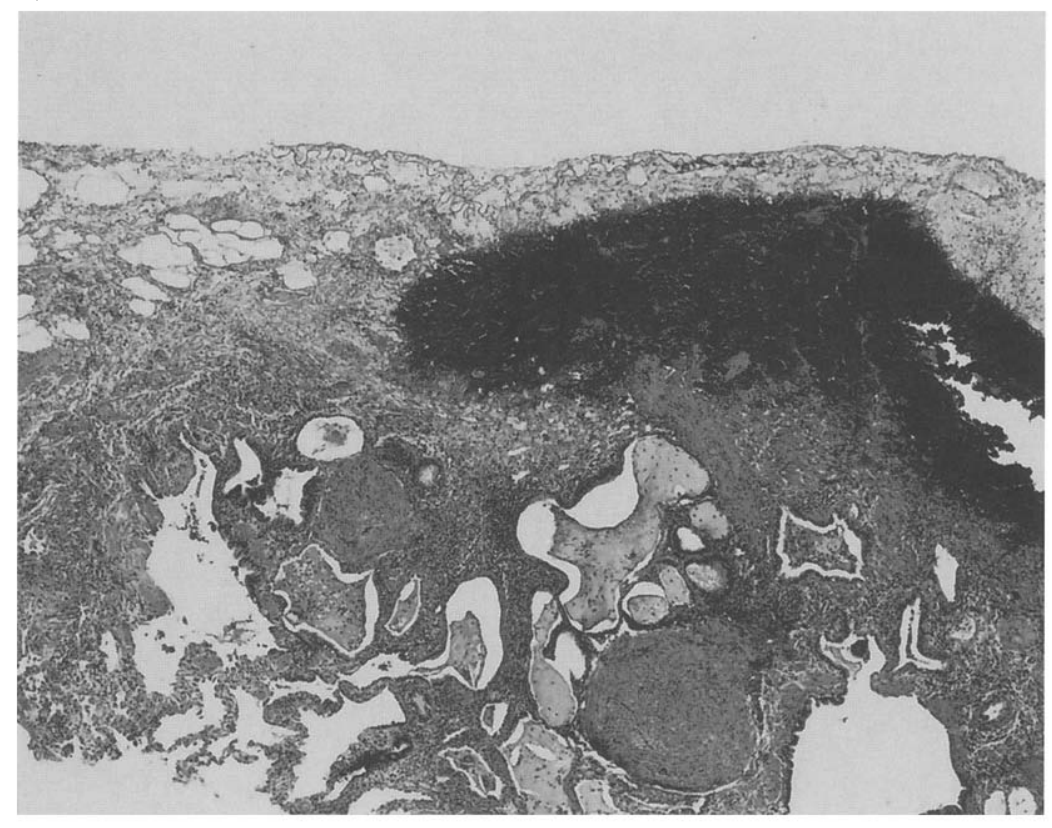

Fig. 1. Traumatic hemorrhage. Biopsy specimen reveals surgically induced subpleural hemorrhage (upper right) in case of idiopathic pulmonary fibrosis.

formalin. Fresh tissue was submitted for culture in appropriately selected cases.

Routinely stained hematoxylin and eosin sections were reviewed in each case. Each biopsy sample was assessed histologically and a final clinicopathologic diagnosis was rendered in correlation with the clinical findings. The following pathologic findings also were semiquantitatively assessed: fresh alveolar or interstitial hemorrhage (Fig. 1) (absent, $<25 \%$ of the tissue affected, $25 \%$ to $50 \%$ of the tissue affected, $>50 \%$ of the tissue affected), compression/ atelectasis (Fig. 2) (absent, mild, severe), traumatic overinflation resembling emphysema (Fig. 2) (absent, present), and neutrophil margination in subpleural small vessels (Fig. 3) (absent, present). The size of tissue specimens was also tabulated.

Clinical findings were obtained from the clinical records. Statistical analyses done were the standard $\chi^{2}$ and Student's $t$ tests.

\section{Results}

The size (longest dimension; mean plus or minus standard deviation) of biopsy specimens determined by measurement at the time of gross examination was $4.1 \pm 2.0 \mathrm{~cm}$ (range 1.2 to $12 \mathrm{~cm}$ ) for patients who underwent VATS-LB and $4.2 \pm 2.1 \mathrm{~cm}(1.5$ to $17 \mathrm{~cm}$ ) for patients who underwent OLB. The difference was not significant.

A specific clinicopathologic diagnosis was made in all 71 patients who underwent VATS-LB. Fifty-one patients had noninfectious inflammatory conditions. This included 15 patients with usual interstitial pneumonia/idiopathic pulmonary fibrosis; 7 with idiopathic bronchiolitis obliterans organizing pneumonia; 7 with hypersensitivity pneumonia (extrinsic allergic alveolitis); 6 with nonclassifiable chronic interstitial pneumonia of unknown cause; 5 with chronic interstitial pneumonia associated with collagen vascular disease; 3 with respiratory bronchiolitis-associated interstitial lung disease; 2 each with desquamative interstitial pneumonia, eosinophilic granuloma, and drug toxicity; and 1 each with eosinophilic pneumonia and chronic passive congestion secondary to underlying cardiac disease. Ten patients had malignant diseases: 5 patients had malignant lymphoma, 3 had bronchioloalveolar adenocarcinoma, and 2 had metastatic adenocarcinoma. Five patients had infections: 2 patients had tuberculosis and one each had cytomegalovirus pneumonia, histoplasmosis, and Pneumocystis carinii pneumonia. One additional patient had allergic bronchopulmonary aspergillosis. Two patients had pulmonary hypertension, one had pulmonary emboli, and one had diffuse alveolar hemorrhage with capillaritis.

Specimens obtained from OLB also were diagnostic in all 42 cases and showed a similar range of abnormalities. Twenty-one patients had noninfectious inflammatory conditions including 9 patients with usual interstitial pneumonia/idiopathic pulmo- 


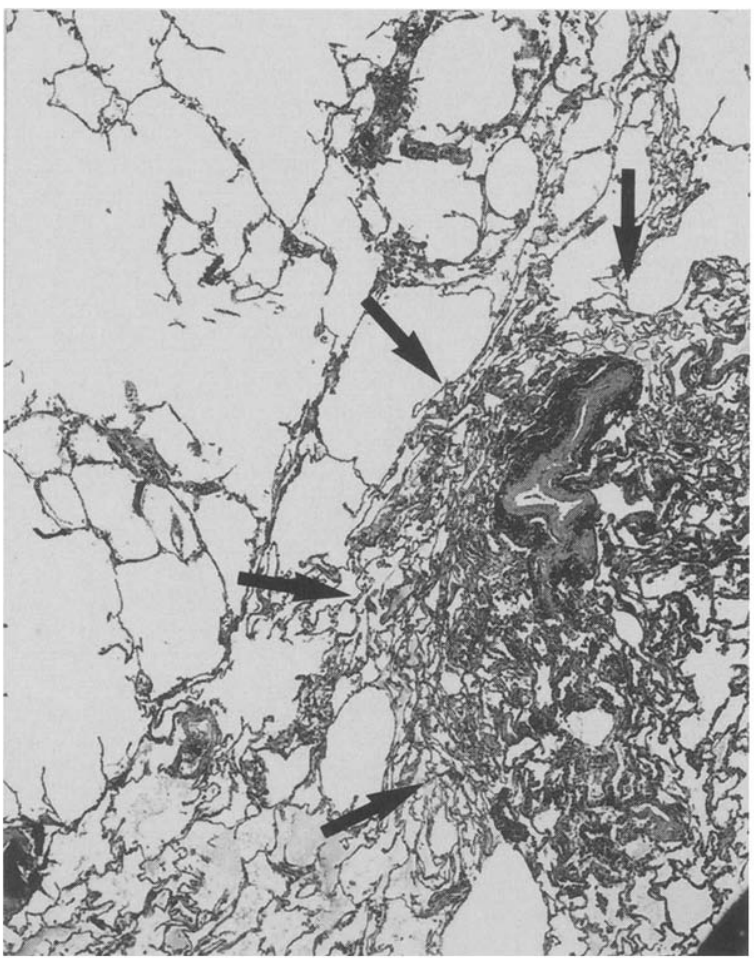

Fig. 2. Surgically induced compression and overinflation artifact. Biopsy specimen reveals compression artifact (arrows) in one region of lung and overinflation of surrounding tissue (upper left).

nary fibrosis, 4 with idiopathic bronchiolitis obliterans organizing pneumonia, 3 with chronic interstitial pneumonia of unknown cause, two each with hypersensitivity pneumonia (extrinsic allergic alveolitis) and drug toxicity, and one with desquamative interstitial pneumonia. Eleven patients had malignant diseases: 6 had malignant lymphoma, three had leukemic infiltrates, and one each had bronchogenic adenocarcinoma and metastatic adenocarcinoma. Nine patients had pulmonary infections: 3 patients had aspergillosis, two each had cytomegalovirus pneumonia and Pneumocystis carinii pneumonia, and one each had herpes pneumonia or tuberculosis. One patient had pulmonary thromboembolic disease.

The pathologic findings interpreted as procedureinduced are summarized in Table $I$. There was no difference between the VATS-LB and the OLB groups in the extent of compression/atelectasis. Not unexpectedly, alveolar or interstitial hemorrhage and neutrophil margination were significantly more common in patients who underwent VATS-LB than in patients who underwent OLB. The extent of overinflation was significantly greater for the OLB group than for the VATS-LB group.

Complications developed in $11(15 \%)$ of 71 patients in the VATS-LB group including 5 patients with prolonged air leakage (more than 10 days); 2 with pneumonia; and 1 each with bleeding, late pneumothorax necessitating readmission, mucus plug necessitating bronchoscopy, and a hypoxic episode necessitating mechanical ventilation. On the other hand, 7 (17\%) of 42 patients in the OLB group had complications including 4 patients with prolonged air leakage (more than 10 days) and 3 with pneumonia. There were $6(8 \%)$ operative deaths in patients who had VATS-LB and $7(17 \%)$ in the OLB group; all of these patients had severe acute interstitial lung disease with preoperative respiratory failure.

\section{Discussion}

Our results underscore the comparative efficacy and safety of VATS-LB in the setting of localized and diffuse lung disease. Thoracoscopic techniques have been used for more than 80 years for the diagnosis of intrathoracic disorders, ${ }^{1}$ but the technique has been expanded with the introduction of miniaturized video cameras and percutaneous stapling devices for pulmonary wedge resections. ${ }^{3}$ VATS-LB is less invasive and less painful than OLB and is associated with less postoperative pulmonary dysfunction. ${ }^{4-6}$

VATS-LB has become a common alternative to OLB for diagnosis of localized and diffuse lung diseases. In only $4 \%$ of our cases ( 3 of 74 ) did technical considerations (pleural adhesions) lead to an open biopsy procedure instead of VATS-LB. We found that the amount of tissue recovered was adequate and the diagnostic yield of VATS-LB was identical to that of OLB: both procedures resulted in a clinicopathologic diagnosis in all cases. Bensard and associates ${ }^{4}$ found similar results when they compared VATS-LB and OLB in 43 patients with interstitial lung diseases: VATS-LB was diagnostic in $95 \%$ of cases versus $100 \%$ for OLB. These authors also concluded that VATS-LB was as safe and effective as OLB in the evaluation of diffuse interstitial lung diseases. ${ }^{4}$

Histologic artifacts related to the surgical procedure itself are common in lung biopsy specimens. ${ }^{7}$ Gaensler and Carrington ${ }^{8}$ studied 502 OLB procedures in patients with chronic interstitial lung disease and found that hemorrhage and atelectasis sometimes interfered with histologic evaluation. They suggested that palpation and clamps should be 


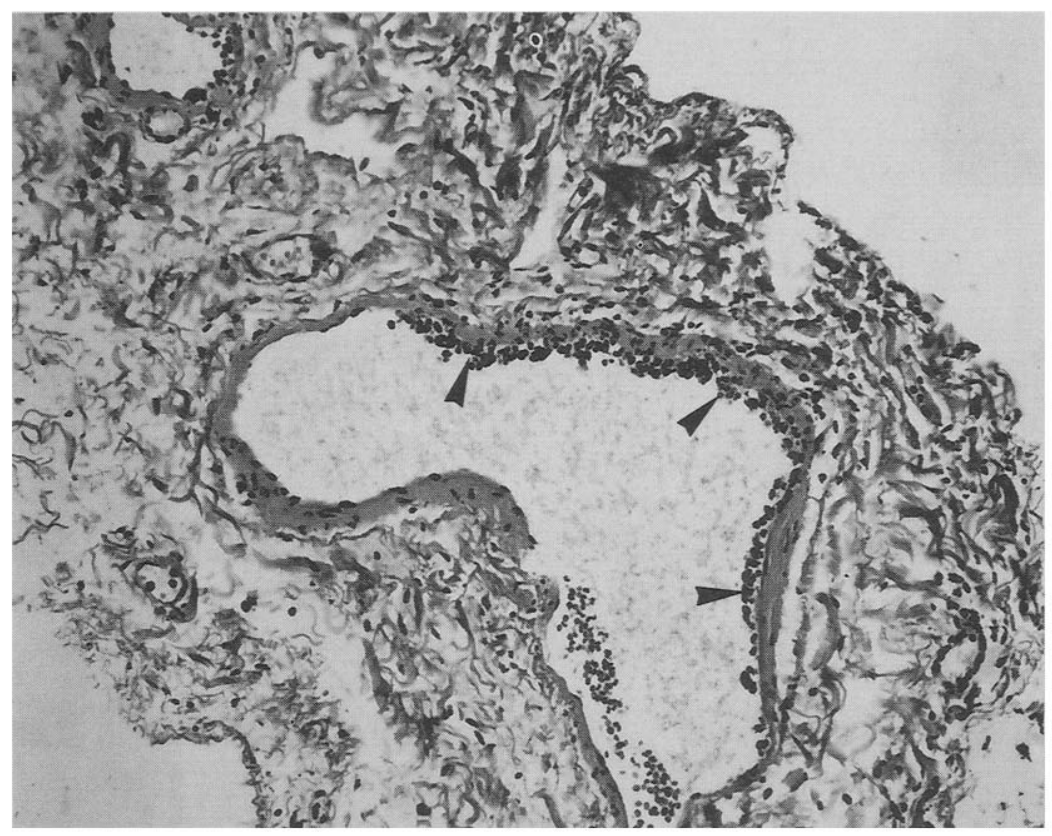

Fig. 3. Neutrophil margination in subpleural vessels. Endothelium is lined by white blood cells (arrowheads), which could be mistaken for vasculitis.

avoided, that the lung wedge should be delineated during full inflation, and that specimens should be fixed immediately. Lung collapse is mandatory during VATS-LB for proper visualization, and therefore it is technically impossible to take the biopsy specimen with the lung fully inflated or to keep the lung inflated when the specimen is being withdrawn through the chest wall.

With any local injury an inflammatory response ensues: leukocytes adhere to endothelium (margination), advance toward an endothelial junction, and emigrate through it and toward chemoattractants emanating from the source of injury. ${ }^{9}$ Margination and fresh hemorrhage are extremely common in surgical specimens, particularly those from tissues that have been extensively manipulated before biopsy or resection, and these changes represent artifacts that pathologists must be familiar with to avoid misdiagnosis. Such changes were common in all our biopsy specimens. However, there were significant differences in the extent of hemorrhage and neutrophil margination between the VATS-LB and OLB groups. These results suggest VATS-LB necessitates more surgical manipulation of the lung than OLB. This is likely because of reduced access and limited visualization.

Despite the fact that both VATS-LB and OLB commonly show artifactual changes and the effects
Table I. Pathologic findings in patients who underwent VATS-LB or OLB

\begin{tabular}{|c|c|c|c|}
\hline \multirow[b]{2}{*}{ Grade } & \multicolumn{2}{|c|}{$\begin{array}{l}\text { Pathologic findings } \\
\text { (\% of cases) }\end{array}$} & \multirow[b]{2}{*}{$\begin{array}{c}p \text { Values } \\
(V A T S-L B \\
v s O L B)\end{array}$} \\
\hline & $\begin{array}{c}\text { VATS-LB } \\
\text { group } \\
\text { (71 cases) }\end{array}$ & $\begin{array}{l}\text { OLB group } \\
\text { (42 cases) }\end{array}$ & \\
\hline \multicolumn{4}{|c|}{$\begin{array}{c}\text { Alveolar or interstitial } \\
\text { hemorrhage }\end{array}$} \\
\hline Absent & 38 & 62 & $<0.025$ \\
\hline$<25 \%$ & 58 & 38 & \\
\hline $25 \%-50 \%$ & 4 & 0 & \\
\hline$>50 \%$ & 0 & 0 & \\
\hline \multicolumn{4}{|c|}{ Compression/atelectasis } \\
\hline Absent & 45 & 60 & NS \\
\hline Mild & 52 & 40 & \\
\hline Severe & 3 & 0 & \\
\hline \multicolumn{4}{|l|}{ Overinflation } \\
\hline Present & 13 & 33 & $<0.025$ \\
\hline \multicolumn{4}{|c|}{ Neutrophil margination } \\
\hline Present & 76 & 57 & $<0.05$ \\
\hline
\end{tabular}

of tissue manipulation (Table I), the real question is whether these changes actually compromise patient care. We do not think they do: a clinicopathologic diagnosis, adequate for patient management and compatible with the initial clinical suspicions, was 
made in every patient. The pathologic changes of margination, hemorrhage, compression/atelectasis, and overinflation are readily recognizable by pathologists and separable from those changes that explain the patient's clinical disease.

\section{REFERENCES}

1. Miller DL, Allen MS, Trastek VF, Deschamps C, Pairolero PC. Videothoracoscopic wedge excision of the lung. Ann Thorac Surg 1992;54:410-4.

2. Ferguson MK. Thoracoscopy for diagnosis of diffuse lung disease. Ann Thorac Surg 1993;56:694-6.

3. Allen MS, Trastek VF, Daly RC, Deschamps C, Pairolero PC. Equipment for thoracoscopy. Ann Thorac Surg 1993;56:620-3.

4. Bensard DD, McIntyre RC, Waring BJ, Simon JS. Comparison of video thoracoscopic lung biopsy to open lung biopsy in the diagnosis of interstitial lung disease. Chest 1993;103:765-70.
5. Donnelly RJ, Page RD, Berrisford RG, Dedeilias PG. Videothoracoscopic surgery. Eur J Cardiothorac Surg 1993;7:281-6.

6. Ferson PF, Landreneau RJ, Dowling RD, et al. Comparison of open versus thoracoscopic lung biopsy for diffuse infiltrative pulmonary disease. J THORAC CARDIOVASC SURG 1993;106:194-9.

7. Colby TV, Yousem SA. Pulmonary histology for the surgical pathologist. Am J Surg Pathol 1988;12:223-39.

8. Gaensler EA, Carrington CB. Open biopsy for chronic diffuse infiltrative lung disease: clinical, roentgenographic, and physiological correlations in 502 patients. Ann Thorac Surg 1980;30:411-26.

9. Cotran RS, Kumar V, Robbins SL. Cellular events. In: Robbins pathologic basis of disease. 4th ed. Philadelphia: WB Saunders, 1989:45-54. 